\title{
Creatinine-To-Cystatin C Ratio as a Marker of Sarcopenia for Identifying Osteoporosis in Male Patients with Type 2 Diabetes Mellitus
}

\section{Huifan Dai}

Second Affiliated Hospital \& Yuying Children's Hospital of Wenzhou Medical University Jing Xu ( 47914057@qq.com )

Second Affiliated Hospital \& Yuying Children's Hospital of Wenzhou Medical University

\section{Research Article}

Keywords: type2 diabetes, osteoporosis, bone mineral density

Posted Date: February 21st, 2022

DOI: https://doi.org/10.21203/rs.3.rs-1364770/v1

License: (c) (1) This work is licensed under a Creative Commons Attribution 4.0 International License.

Read Full License 


\section{Abstract \\ Background}

Type 2 diabetes mellitus (T2DM) is associated with an increased incidence rate of sarcopenia and osteoporosis. Serum creatinine-to-cystatin $\mathrm{C}$ ratio (CCR) is a novel and simple tool which can be used as an index of sarcopenia. This study aims to investigate the association between CCR and osteoporosis and bone mineral density (BMD) in T2DM patients.

\section{Methods}

Recruited 166 females and 252 males with T2DM patients. Collect general information, BMD data and laboratory data. The correlation between CCR, BMD, bone metabolism markers and osteoporosis was discussed by spearman correlation, receiver-operating characteristic (ROC) curve analysis and multiple regression analysis.

\section{Results}

Spearman correlation analysis showed that CCR in male patients was positively correlated with ASM/height ${ }^{2}$ and BMD $(r=215-0.381, P<0.01)$, but the female group did not show such relationship. In multivariate regression analysis, it was found that there was a significant correlation between CCR and BMD of total lumbar spine, hip and femoral neck in male patients. ROC curve showed that the optimal cut-off value of CCR for predicting osteoporosis in male patients was 6.73 with the sensitivity of $88 \%$ and specificity of $63 \%$.

\section{Conclusion}

In male T2DM patients, CCR was negatively correlated with osteoporosis and positively correlated with BMD.

\section{Introduction}

Fractures and osteoporosis are on the rise in older people, which can result in disability, decline in quality of life, and even death ${ }^{1}$. Patients with Type 2 diabetes mellitus (T2DM) are generally more likely to suffer from fracture than the general population ${ }^{2,3}$. Previous research had analyzed and reported risk factor associated with osteoporosis in the general population ${ }^{4}$. However, it is important to explore the risk factors for osteoporosis, which may differ from due to metabolic disorders.

Aging can result in osteoporosis, but it can also lead to a decreased muscle mass. Low mass of muscle is called sarcopenia. The relationship between the sarcopenia and osteoporosis in chronic obstructive 
pulmonary disease ${ }^{5}$, chronic kidney disease ${ }^{6}$, primary biliary cholangitis ${ }^{7}$ has been well developed. We have also confirmed that mass of skeletal muscle is independently associated with osteoporosis in type 2 diabetes ${ }^{8}$. However, traditional skeletal muscle mass inspection methods, such as dual-energy-X-ray absorption method, are complex and costly.

Serum creatinine is a metabolic waste produced by skeletal muscle. Cystatin $\mathrm{C}$ can be produce by nucleated cells in the body at a constant production rate, which can only be removed through glomerular filtration. Some studies supported that the creatinine-to-cystatin $\mathrm{C}$ ratio (CCR) can be used as a biomarker of skeletal muscle mass. Takafumi et al. suggested that CCR is an inexpensive and simple method of measuring sarcopenia in patients with type 2 diabetes $^{9}$. However, whether this ratio can be regarded as a predictor of osteoporosis in type2 diabetes has not been studied. Therefore, this study aims to explore the relationship between CCR, osteoporosis and BMD in patients with T2DM.

\section{Methods}

\section{Subjects}

The study included 418 T2DM patients (age>50 years old) in China. The subjects were evaluated or treated for T2DM at the Second Affiliated Hospital of Wenzhou Medical University and Yuying Children's Hospital from January 2020 to March 2021. Inclusion criteria included normal renal function (glomerular filtration rate $>60 \mathrm{ml} / \mathrm{min}$, creatine $\leq 115 \mu \mathrm{mmol} / \mathrm{L}$ ). Exclusion criteria included (a)malignant tumors and severe heart or liver diseases; (b) diagnosis of adrenal, gonadal, parathyroid, pituitary and thyroid diseases; (c) long-term use of calcium, vitamin D, or other drugs that influence bone metabolism; (d) patients who are bedridden for a long time; and (e) patients who lack available information. This study was approved by the Ethics Committee of the Second Affiliated Hospital of Wenzhou Medical University (No. LCKY2020-03, date: January 2020), and obtained the written informed consent of all participants according to the Declaration of Helsinki.

\section{Health history and clinical assessment}

Their height and weight without wearing shoes were measured. BMI was calculated by dividing weight $(\mathrm{Kg})$ by the square of height $\left(\mathrm{m}^{2}\right)$. The duration of diabetes was calculated in years, which ranged from the diagnosis of type 2 diabetes in the patient's medical record to our BMD measurements and blood test.

\section{Biochemical parameters}

Serum samples are collected at 6 am after fasting at night (at least $8 \mathrm{~h}$ ). Standard laboratory methods were used to measure serum lipid metabolism indicators, including high density lipoprotein cholesterol (HDL-C), total cholesterol (TC), low density lipoprotein cholesterol (LDL-C), triglyceride (TG) levels; glucose metabolism indicators, including (FBG) fasting blood glucose, (HbA1c) glycosylated hemoglobin; Bone metabolism indicators, including $\beta$-CTX, PINP, 25-hydroxy-vitamin (25(OH)D) and parathyroid hormone 
(PTH). Besides, laboratory markers such as serum Cystatin C, creatinine (Cr), calcium (Ca), uric and albumin were recorded.

\section{BMD measurement}

Whole-body dual-energy X-ray absorptiometry (DXA; USA, Hologic Discovery) was used to measure body fat content, trunk muscle mass and BMD of the lumbar spine, femur neck, total hip and skeletal muscle mass index (ASMI). The calculation formula of ASMI is as follows: appendicular skeletal muscle mass (ASM)/height ${ }^{2}{ }^{10}$. The T scores was calculated using the DXA database. Normal bone mass was defined as a $T$ value $>-1.0$; osteoporosis, $T$ value $<-2.5$ and osteopenia, $-1.0>T$ value $>-2.5^{11}$.

\section{Statistical analysis}

The proportion was used to describe categorical variables and mean \pm standard deviation to describe continuous variables. The means and proportions were compared using the one-way ANOVA analysis and Pearson's $\chi 2$-test. Spearman coefficient was used to analyze CCR, BMD and bone metabolism index. Since there was no correlation between CCR and BMD in female group, multivariate analysis was only conducted in the male group. The correlation between BMD of femoral neck, lumbar spine, hip and other variables was analyzed by multivariate linear regression analysis. Odds rations (ORs) and $95 \%$ confidence intervals (Cls) was calculated by Logistic analysis. We plotted the receiver operating characteristic (ROC) curve and calculated the area under the curve (AUC) to assess the clinical value of CCR in predicting osteoporosis

\section{Results}

\section{Basic Characteristics}

Patient characteristics are shown in Table 1. The retrospective study included 418 T2DM patients with a BMI of $24.5 \pm 3.2 \mathrm{~kg} / \mathrm{m}^{2}$ and an average age of $60.8 \pm 7.3$ years old. The BMD of the hip, femur neck and lumbar spine were higher in males $(1.007$ vs $0.903,0.941$ vs $0.826,1.194$ vs 1.012 , all $P<0.01)$ compared with females. The levels of HDL-c, FBG, HbA1c, LDL-c, PINP, $\beta-C T X$ and the incidence of osteoporosis in males were significantly lower than those in females. The levels of creatinine, cystatin C, CCR and ASM/height ${ }^{2}$ were significantly higher in males than those in females. 
Table 1

Patient characteristics, stratified by sex.

\begin{tabular}{|c|c|c|c|c|}
\hline & $\begin{array}{l}\text { Total patients } \\
(n=418)\end{array}$ & $\begin{array}{l}\text { male patients } \\
(n=252)\end{array}$ & $\begin{array}{l}\text { female patients } \\
(n=166)\end{array}$ & $\mathbf{P}$ \\
\hline Age, years & $60.8 \pm 7.3$ & $59.8 \pm 7.4$ & $62.2 \pm 6.9$ & 0.001 \\
\hline Diabetic duration, years & $9.2 \pm 7.1$ & $8.5 \pm 6.9$ & $10.2 \pm 7.3$ & 0.019 \\
\hline Systolic blood pressure, $\mathrm{mmHg}$ & $142.7 \pm 25.1$ & $141.9 \pm 24.3$ & $143.9 \pm 26.4$ & 0.427 \\
\hline Diastolic blood pressure, $\mathrm{mmHg}$ & $85.2 \pm 41.7$ & $87.4 \pm 53.3$ & $81.9 \pm 7.6$ & 0.186 \\
\hline $\mathrm{BMl}, \mathrm{Kg} / \mathrm{m} 2$ & $24.5 \pm 3.2$ & $24.8 \pm 2.9$ & $24.0 \pm 3.6$ & 0.016 \\
\hline \multicolumn{5}{|l|}{ Laboratory findings } \\
\hline FBG, mmol/L & $6.6 \pm 1.9$ & $6.5 \pm 1.9$ & $6.8 \pm 1.9$ & 0.062 \\
\hline $\mathrm{HbA1c}, \mathrm{mmol} / \mathrm{L}$ & $9.1 \pm 2.0$ & $8.9 \pm 2.0$ & $9.4 \pm 2.1$ & 0.046 \\
\hline $\mathrm{TC}, \mathrm{mmol} / \mathrm{L}$ & $4.55 \pm 1.25$ & $4.48 \pm 1.22$ & $4.78 \pm 1.25$ & 0.450 \\
\hline $\mathrm{TG}, \mathrm{mmol} / \mathrm{L}$ & $1.89 \pm 1.46$ & $1.94 \pm 1.46$ & $1.82 \pm 1.48$ & 0.002 \\
\hline $\mathrm{HDL}-\mathrm{c}, \mathrm{mmol} / \mathrm{L}$ & $1.06 \pm 0.29$ & $0.98 \pm 0.26$ & $1.16 \pm 0.30$ & $<0.001$ \\
\hline LDL-c, mmol/L & $2.76 \pm 1.02$ & $2.66 \pm 1.00$ & $2.91 \pm 1.03$ & 0.022 \\
\hline Albumin, g/L & $40.0 \pm 3.4$ & $40.3 \pm 3.4$ & $39.6 \pm 3.5$ & 0.084 \\
\hline Creatinine, $\mu \mathrm{mol} / \mathrm{L}$ & $62.7 \pm 31.2$ & $69.5 \pm 30.8$ & $52.3 \pm 28.9$ & $<0.001$ \\
\hline Cystatin C, mg/L & $0.99 \pm 0.34$ & $1.02 \pm 0.33$ & $0.95 \pm 0.34$ & 0.023 \\
\hline CCR & $7.6 \pm 1.6$ & $8.2 \pm 1.6$ & $6.7 \pm 1.3$ & $<0.001$ \\
\hline Serum uric, $\mu \mathrm{mmol} / \mathrm{L}$ & $328.3 \pm 92.7$ & $349.3 \pm 87.0$ & $298.7 \pm 92.7$ & $<0.001$ \\
\hline PTH, pg/ml & $38.6 \pm 15.9$ & $40.1 \pm 16.6$ & $36.4 \pm 14.7$ & 0.020 \\
\hline PINP, ng/ml & $40.8 \pm 19.5$ & $37.8 \pm 17.7$ & $45.4 \pm 21.1$ & $<0.001$ \\
\hline$\beta-C T X, n g / m l$ & $0.46 \pm 0.24$ & $0.43 \pm 0.22$ & $0.50 \pm 0.27$ & 0.004 \\
\hline $25(\mathrm{OH}) \mathrm{D}, \mathrm{ng} / \mathrm{ml}$ & $22.55 \pm 8.01$ & $23.43 \pm 8.34$ & $21.20 \pm 7.30$ & 0.006 \\
\hline Calcium, mmol/L & $2.24 \pm 0.11$ & $2.24 \pm 0.11$ & $2.25 \pm 0.11$ & 0.593 \\
\hline ASM/height ${ }^{2}, \mathrm{~g} / \mathrm{cm}^{2}$ & $6.68 \pm 1.26$ & $7.18 \pm 1.13$ & $5.91 \pm 1.05$ & $<0.001$ \\
\hline \multicolumn{5}{|c|}{$\begin{array}{l}\text { Values are mean SD or number (\%). P< } 0.05 \text { was deemed significant (comparison between men and } \\
\text { women group). BMI, body mass index; FBG, fasting blood glucose; HbA1c, glycosylated hemoglobin; } \\
\text { TC, total cholesterol; TG, triglyceride; HDL-C, High density lipoprotein cholesterol; LDL-C, Low density } \\
\text { lipoprotein cholesterol; PTH, parathyroid hormone; } 25(\mathrm{OH}) \mathrm{D} \text {, 25-hydroxy-vitamin; CCR, Creatinine-to- } \\
\text { Cystatin C ratio }\end{array}$} \\
\hline
\end{tabular}




\begin{tabular}{|c|c|c|c|c|}
\hline & $\begin{array}{l}\text { Total patients } \\
(n=418)\end{array}$ & $\begin{array}{l}\text { male patients } \\
(n=252)\end{array}$ & $\begin{array}{l}\text { female patients } \\
(n=166)\end{array}$ & $\mathbf{P}$ \\
\hline \multicolumn{5}{|l|}{ BMD } \\
\hline Total lumbar, $\mathrm{g} / \mathrm{cm}^{2}$ & $1.122 \pm 0.206$ & $1.194 \pm 0.184$ & $1.012 \pm 0.187$ & $<0.001$ \\
\hline Femur neck, g/cm² & $0.896 \pm 0.149$ & $0.941 \pm 0.142$ & $0.826 \pm 0.131$ & $<0.001$ \\
\hline Total hip, $\mathrm{g} / \mathrm{cm}^{2}$ & $0.966 \pm 0.151$ & $1.007 \pm 0.141$ & $0.903 \pm 0.144$ & $<0.001$ \\
\hline Osteoporosis & $20.6 \%$ & $11.1 \%$ & $34.9 \%$ & $<0.001$ \\
\hline \multicolumn{5}{|c|}{$\begin{array}{l}\text { Values are mean SD or number (\%). P<0.05 was deemed significant (comparison between men and } \\
\text { women group). BMI, body mass index; FBG, fasting blood glucose; HbA1c, glycosylated hemoglobin; } \\
\text { TC, total cholesterol; TG, triglyceride; HDL-c, High density lipoprotein cholesterol; LDL-C, Low density } \\
\text { lipoprotein cholesterol; PTH, parathyroid hormone; } 25(\mathrm{OH}) \mathrm{D} \text {, 25-hydroxy-vitamin; CCR, Creatinine-to- } \\
\text { Cystatin C ratio }\end{array}$} \\
\hline
\end{tabular}

\section{Comparison of CCR, and other biochemical and clinical indicators of T2DM patients with normal BMD values, osteoporosis, osteopenia}

Based on the BMD T value measured by DXA, T2DM patients were divided into normal BMD, osteoporosis and osteopenia groups (Table 2). Compared to normal BMD group, BMI, albumin, creatinine, uric and $\mathrm{ASM} /$ height $^{2}$ were significantly reduced and age, diabetic duration, FBG, cystatin C, PINP and $\beta$-CTX levels significantly increased in the osteoporosis and osteopenia groups. The BMD of the total hip, femur neck and total lumbar spine in the osteoporosis and osteopenia groups were significantly decreased. Compared with the normal BMD group, the level of CCR in osteoporosis group was lower. 
Table 2

Comparison of various parameters of T2DM patients with differently BMD T value.

\begin{tabular}{|c|c|c|c|}
\hline & Normal $(n=193)$ & Osteopenia $(n=140)$ & Osteoporosis $(n=85)$ \\
\hline Age, years & $58.4 \pm 6.6$ & $61.8 \pm 7.0^{*}$ & $64.5 \pm 7.2^{\star \#}$ \\
\hline Diabetic duration, years & $8.2 \pm 6.9$ & $9.6 \pm 7.1^{*}$ & $10.7 \pm 9.2^{*}$ \\
\hline Systolic blood pressure, $\mathrm{mmHg}$ & $140.7 \pm 25.0$ & $145.5 \pm 25.2$ & $142.8 \pm 25.0$ \\
\hline Diastolic blood pressure, $\mathrm{mmHg}$ & $84.1 \pm 8.6$ & $88.6 \pm 71.3$ & $82.3 \pm 6.9$ \\
\hline $\mathrm{BMI}, \mathrm{Kg} / \mathrm{m}^{2}$ & $25.2 \pm 3.2$ & $24.0 \pm 3.0^{*}$ & $23.5 \pm 3.3^{*}$ \\
\hline \multicolumn{4}{|l|}{ Laboratory findings } \\
\hline $\mathrm{FBG}, \mathrm{mmol} / \mathrm{L}$ & $6.4 \pm 1.7$ & $6.7 \pm 2.0^{\star}$ & $6.7 \pm 2.1^{*}$ \\
\hline $\mathrm{HbA} 1 \mathrm{c}, \mathrm{mmol} / \mathrm{L}$ & $9.1 \pm 2.1$ & $9.1 \pm 1.9$ & $9.1 \pm 2.1$ \\
\hline $\mathrm{TC}, \mathrm{mmol} / \mathrm{L}$ & $4.5 \pm 1.3$ & $4.5 \pm 1.2$ & $4.7 \pm 1.3$ \\
\hline TG, mmol/L & $2.0 \pm 1.8$ & $2.0 \pm 1.3$ & $1.6 \pm 0.9^{\star \#}$ \\
\hline $\mathrm{HDL}-\mathrm{c}, \mathrm{mmol} / \mathrm{L}$ & $1.0 \pm 1.3$ & $1.1 \pm 0.3$ & $1.1 \pm 0.3^{\star \#}$ \\
\hline LDL-C, mmol/L & $2.7 \pm 1.0$ & $2.7 \pm 1.0$ & $3.0 \pm 1.1$ \\
\hline Albumin, $\mathrm{g} / \mathrm{L}$ & $40.5 \pm 3.3$ & $40.3 \pm 3.5$ & $38.6 \pm 3.2^{\star \#}$ \\
\hline Creatinine, $\mu \mathrm{mol} / \mathrm{L}$ & $65.9 \pm 34.1$ & $62.9 \pm 27.0^{*}$ & $55.0 \pm 29.8^{* \#}$ \\
\hline Cystatin C & $0.96 \pm 0.34$ & $1.02 \pm 0.32^{*}$ & $1.04 \pm 0.33^{*}$ \\
\hline CCR & $8.3 \pm 1.6$ & $7.4 \pm 1.3^{*}$ & $6.3 \pm 1.3^{\star \#}$ \\
\hline Uric, $\mu \mathrm{mmol} / \mathrm{L}$ & $346.3 \pm 91.7$ & $325.1 \pm 93^{*}$ & $297.9 \pm 86.5^{\star \#}$ \\
\hline PTH, pg/ml & $36.9 \pm 14.7$ & $38.9 \pm 15.6$ & $39.1 \pm 16.7$ \\
\hline PINP, ng/ml & $37.4 \pm 17.7$ & $41.9 \pm 17.6^{*}$ & $46.7 \pm 24.4^{*}$ \\
\hline$\beta-C T X, n g / m l$ & $0.41 \pm 0.20$ & $0.47 \pm 0.25^{\star}$ & $0.53 \pm 0.29^{\star}$ \\
\hline $25(\mathrm{OH}) \mathrm{D}, \mathrm{ng} / \mathrm{ml}$ & $22.9 \pm 7.6$ & $22.9 \pm 8.9$ & $21.0 \pm 7.3^{*}$ \\
\hline Calcium, mmol/L & $2.25 \pm 0.11$ & $2.24 \pm 0.10$ & $2.24 \pm 0.11^{*}$ \\
\hline ASM $/$ height $^{2}, \mathrm{Kg} / \mathrm{m}^{2}$ & $7.1 \pm 1.3$ & $6.4 \pm 0.9^{\star}$ & $6.2 \pm 1.3^{* \#}$ \\
\hline BMD & & & \\
\hline
\end{tabular}




\begin{tabular}{|llll|}
\hline & Normal $(\mathrm{n}=193)$ & Osteopenia $(\mathrm{n}=140)$ & Osteoporosis $(\mathrm{n}=\mathbf{8 5})$ \\
\hline Total lumbar, $\mathrm{g} / \mathrm{cm}^{2}$ & $1.257 \pm 0.157$ & $1.051 \pm 0.147^{*}$ & $0.932 \pm 0.176^{* \#}$ \\
\hline Femur neck, $\mathrm{g} / \mathrm{cm}^{2}$ & $1.003 \pm 0.110$ & $0.826 \pm 0.092^{*}$ & $0.766 \pm 0.128^{\star \#}$ \\
\hline Total hip, $\mathrm{g} / \mathrm{cm}^{2}$ & $1.070 \pm 0.112$ & $0.899 \pm 0.106^{*}$ & $0.839 \pm 0.130^{* \#}$ \\
\hline
\end{tabular}

\section{Spearman correlations analysis between CCR, BMD and bone metabolism index}

The Spearman correlation coefficients ( $r$ ) of CCR and BMD of lumbar, femur neck, hip, PTH, serum Ca, ASM $/$ height $^{2}$ were $0.381,0.302,0.323,-0.141,0.181,0.215$ in male groups, respectively. However, CCR was not correlated with the BMD in female groups (Table 3 ).

Table 3

Correlation analysis between CCR, BMD and bone metabolism markers.

\begin{tabular}{|lllll|}
\hline & Male & \multicolumn{3}{l|}{ female } \\
\hline Variables & $r$ & $\mathrm{P}$ & $\mathrm{r}$ & $\mathrm{P}$ \\
\hline Total lumbar BMD & 0.381 & $<0.001$ & 0.107 & 0.192 \\
\hline Total hip BMD & 0.302 & $<0.001$ & 0.013 & 0.669 \\
\hline Femur neck BMD & 0.323 & $<0.001$ & 0.029 & 0.721 \\
\hline PTH & -0.141 & 0.033 & -0.137 & 0.039 \\
\hline PINP & -0.087 & 0.194 & -0.014 & 0.835 \\
\hline B-CTX & -0.026 & 0.693 & -0.015 & 0.822 \\
\hline 25(OH)D & 0.050 & 0.451 & -0.010 & 0.877 \\
\hline Calcium & 0.181 & 0.008 & 0.226 & 0.001 \\
\hline ASM/height ${ }^{2}$ & 0.215 & 0.002 & 0.085 & 0.335 \\
\hline
\end{tabular}

\section{Linear regression analysis for BMD in male group.}

Age, $\mathrm{BMI}$ and $\mathrm{HbA} 1 \mathrm{c}$ had a significant effect on osteoporosis prevalence. Accordingly, we performed subgroup analyses based on BMI, age and $\mathrm{HbA} 1 \mathrm{c}$ (Table 4). After adjusting SBP, DBP, age, duration of diabetic, HDL-C, TG, LDL-C, TC, creatinine, FBG, uric and HbA1c, CCB had an independent relationship with the BMD of the femoral neck, lumbar spine, and hip in the male group. 
Table 4

Creatinine/ Cystatin $\mathrm{C}$ was independent association with BMD based on the cross-categorization of $\mathrm{BMI}$, age and $\mathrm{HbA} 1 \mathrm{c}$ in male patients.

\begin{tabular}{|lllllll|}
\hline & \multicolumn{2}{l}{ Total lumbar } & \multicolumn{2}{l}{ Femur neck } & \multicolumn{2}{l|}{ Total hip } \\
\hline male & $\beta$ & $\mathrm{P}$ & $\beta$ & $\mathrm{P}$ & $\beta$ & $\mathrm{P}$ \\
\hline $\mathrm{BMl}<24 \mathrm{~kg} / \mathrm{m}^{2}$ & 0.206 & 0.038 & 0.229 & 0.047 & 0.200 & 0.212 \\
\hline $\mathrm{BMl} \geq 24 \mathrm{~kg} / \mathrm{m}^{2}$ & 0.271 & 0.015 & 0.252 & 0.044 & 0.212 & 0.049 \\
\hline $\mathrm{Age}<65$ years & 0.285 & 0.012 & 0.269 & 0.020 & 0.271 & 0.020 \\
\hline $\mathrm{Age} \geq 65$ years & 0.388 & 0.003 & 0.338 & 0.011 & 0.330 & 0.014 \\
\hline $\mathrm{HbA} 1 \mathrm{c}<9.0 \%$ & 0.276 & 0.018 & 0.204 & 0.046 & 0.229 & 0.049 \\
\hline $\mathrm{HbA} 1 \mathrm{c} \geq 9.0 \%$ & 0.320 & 0.020 & 0.236 & 0.037 & 0.267 & 0.044 \\
\hline
\end{tabular}

\section{Logistic regression analyses of male osteoporosis.}

Logistic regression analysis was used to test the association between CCR and osteoporosis in male group (Table 5). Although the odds ratio decreased after adjusting SBP, DBP, diabetic duration, age, LDL-C, HDL-c, TG, TC, albumin, FBG, creatinine, HbA1c, uric, the correlation between CCR and osteoporosis remained significant (odd ratio $=0.336, \mathrm{P}<0.05$ ). 
Table 5

Logistic regression analysis for osteoporosis in male patients.

\begin{tabular}{|lllll|}
\hline & $\mathbf{R}$ & $95 \%$ Cl & S.E & P \\
\hline age & 0.992 & $0.902-1.092$ & 0.049 & 0.876 \\
\hline Diabetic duration & 1.015 & $0.920-1.120$ & 0.050 & 0.765 \\
\hline BMI & 0.777 & $0.574-0.954$ & 0.155 & 0.045 \\
\hline TC & 0.936 & $0.099-8.868$ & 1.147 & 0.954 \\
\hline TG & 1.168 & $0.365-3.740$ & 0.594 & 0.794 \\
\hline HDL-c & 1.859 & $0.057-10.350$ & 1.776 & 0.727 \\
\hline LDL-c & 0.981 & $0.115-8.368$ & 1.094 & 0.986 \\
\hline Albumin & 0.835 & $0.645-1.080$ & 0.131 & 0.169 \\
\hline Uric & 0.998 & $0.989-1.007$ & 0.005 & 0.613 \\
\hline HbA1c & 1.109 & $0.729-1.689$ & 0.214 & 0.628 \\
\hline FPG & 0.791 & $0.470-1.330$ & 0.265 & 0.376 \\
\hline Creatinine/ Cystatin C & 0.336 & $0.172-0.657$ & 0.120 & 0.001 \\
\hline $\begin{array}{l}\text { Abbreviations: BMI, body mass index; TC, total cholesterol; TG, triglyceride; HDL-c, High density } \\
\text { lipoprotein cholesterol; LDL-c, Low density lipoprotein cholesterol; FBG, fasting blood glucose; HbA1c, } \\
\text { glycosylated hemoglobin }\end{array}$ & & & & \\
\hline
\end{tabular}

\section{Prognostic Value Of Ccr}

ROC curve analysis of the impact of CCR on the diagnosis of osteoporosis in male group (Fig. 1). The area under ROC curve was 0.788 . The optimal cut-off value of CCR for predicting osteoporosis was 6.73 with the sensitivity of $88 \%$ and the specificity of $63 \%$.

\section{Discussion}

As far as we know, this study is the first to investigate the relationship between CCR, osteoporosis and BMD in patients in T2DM patients. The main finding of this study is that in male T2DM patients, CCR is positively correlated with BMD, at total hip, lumbar and femur neck, and negatively correlated with osteoporosis.

Muscle breakdown and metabolism can produce creatinine, which is proportional to muscle mass. Therefore, the serum creatinine levels are considered a potential indicator reflecting the muscle mass throughout the body. However, because renal function affects its level, the marker is not practical in practice. Instead, Cystatin $\mathrm{C}$ is not affected by muscle mass throughout the body because it is produced 
in all nucleated cells and is reported to be another sign of glomerular filtration. In recent years, CCR has been proposed as a new marker for skeletal muscle mass ${ }^{12-14}$.

The association between CCR and sarcopenia has been established among the subjects with chronic obstructive pulmonary disease ${ }^{15}$, advanced cancer ${ }^{16}$, obstructive coronary artery disease ${ }^{17}$ and healthy subjects $^{18}$. Takafumi et al. recommend that CCR as a practical screening index for sarcopenia in patients with type2 diabetes ${ }^{9}$. Our findings are consistent with previous studies. It is found that CCR was positively associated with ASM/height ${ }^{2}$ in male group $(r=0.215, P=0.002)$, but the female group did not show such relationship.

Reduced muscle mass can result in the worsening of insulin sensitivity, which in turn can result in diabetes ${ }^{19}$. Diabetes and other systemic diseases caused by sarcopenia can lead to both abnormal bone metabolism and muscle loss ${ }^{20}$. Our results suggest that low CCR is an important risk factor for osteoporosis and decreased BMD in male T2DM patients. To our knowledge, this is the first study showing a direct correlation between CCR, osteoporosis and BMD in T2DM.

Further subgroup analysis considering $\mathrm{BMI}$, age, $\mathrm{HbA} 1 \mathrm{c}$ showed that, among the male patients with higher BMl, older age and higher glycosylated hemoglobin levels, the association between CCR and bone mineral density was higher than that in the control group. Accordingly, for the above patients, CCR may be a simple predictor of BMD in male patients with T2DM.

CCR is an important component of sarcopenia and a predictor of various adverse health outcomes. Lu et al. found that subjects with low CCR is a risk factor for adverse cardiovascular events in patients with obstructive coronary artery disease ${ }^{9}$. Liu et al. suggested that low CCR is a risk factor for long-term poor prognosis and 30-day mortality and in Acute Ischemic Stroke Patients ${ }^{21}$. Qiu et al. reported that low CCR can lead to increased risk of diabetes ${ }^{22}$. Therefore, it will be valuable to identify persons with sarcopenia in clinical practice.

In our study, CCR was negatively associated with osteoporosis in male group ( $O R=0.336, P=0.001)$, but the female group did not show such relationship. This has been confirmed in previous studies that male have a closer relationship between bone mass and muscle mass than in females ${ }^{23-25}$. In males, muscle and bone changes are controlled by elevated IGF-1 and testosterone level, which leads to an increase in muscle mass and strength, while in females, higher levels of estrogen cause bone mass to tend to muscle growth faster ${ }^{23,24}$. Aging leads to loss of muscle and bone in females and males; the relationship between bone and muscle mass is affected by sex differences in bone and muscle mass loss rate ${ }^{25}$. In particular, the age-related declines in testosterone and IGF-1 levels may lead to the loss of muscle and bone in males, while the absolute level and decline of testosterone in females are much lower, and the mass of muscle can be relatively preserved.

Compared with traditional detection methods such as $\mathrm{CT}$, dual-energy-X-ray absorptiometry and bioimpedance analysis, CCR detection only requires blood sampling, which is easy to be analyzed, and 
does not require the patients to bear the high costs of detection. As a result, CCR is more acceptable. In addition, the CCR results are easy to understand and intuitive. Therefore, it is more convenient for clinicians to use the CCR to assess sarcopenia.

This study has several limitations. First of all, we do not know the causal relationship between osteoporosis and the CCR because of the cross-sectional design. Secondly, we do not consider the dietary intake of meat, or drugs, such as cimetidine, determination of exercise. These factors may alter creatinine levels and may affect CCR. Thirdly, the concentrations of serum cystatin $\mathrm{C}$ and creatinine is significantly affected by renal function, suggesting that the CCR may not be suitable for identifying osteoporosis in patients with abnormal renal function, and the prevalence of osteoporosis in patients with chronic kidney diseases is relatively high ${ }^{26}$.

\section{Conclusion}

In summary, we report the first study that lower CCR is closely related to osteoporosis and low BMD in male T2DM patients. When the diagnostic cut-off value of male CCR is 6.73 , the risk of osteoporosis increase, with a sensitivity of $88 \%$ and a specificity of $63 \%$.

\section{Abbreviations}

CCR, Creatinine-to-cystatin C ratio; BMD, Bone Mineral Density; FBG, fasting blood glucose; BMI, body mass index; HbA1c, glycosylated hemoglobin; 25(OH)D, 25-hydroxy-vitamin; PTH, parathyroid hormone.

\section{Declarations}

\section{Funding}

Zhejiang Provincial Department of Health(2016KYB194), General Research Project of Zhejiang Provincial Education Department(Y201534290), Wenzhou Science and Technology Bureau(Y20120163).

\section{Ethics approval and consent to participate}

This study was approved by the Ethics Committee of the Second Affiliated Hospital of Wenzhou Medical University (No. LCKY2020-03, date: January 2020), and obtained the written informed consent of all participants according to the Declaration of Helsinki.

\section{Consent for publication}

Not applicable.

\section{Availability of data and materials}


The data that support the findings of this study are available from Institutional Review Board of the second affiliated hospital and Yuying Children's Hospital of Wenzhou Medical University but restrictions apply to the availability of these data, which were used under license for the current study, and so are not publicly available. Data are however available from the authors upon reasonable request and with permission of Institutional Review Board of the Second Affiliated Hospital and Yuying Children's Hospital of Wenzhou Medical University.

\section{Competing interests}

The authors declare that they have no competing interests.

\section{Authors' contributions}

HFD - study concept and design; preparation, review and approval of manuscript. JX - data collection and interpretation; preparation, review and approval of manuscript.

\section{Acknowledgements}

The authors thank research staff, all subjects, and students who participated in this study.

\section{References}

1. Lin $X$, Xiong D, Peng YQ, et al. Epidemiology and management of osteoporosis in the People's Republic of China: current perspectives. Clin Interv Aging. 2015;10: 1017-1033.

2. Janghorbani M, Van Dam RM, Willett WC, Hu FB. Systematic review of type 2 and diabetes type 1 mellitus and fracture. Journal American epidemiology. 2007;166(5): 495-505.

3. Vestergaard P. Discrepancies in bone mineral density and fracture risk in patients with type 2 and type 1 diabetes-a meta-analysis. Osteoporosis international: a journal cooperation in established with the Foundation European for National Osteoporosis and the Osteoporosis Foundation of the USA. 2017;18(4): 416-444.

4. Holm JP, Hyldstrup L, Jensen JB. Time trends in osteoporosis risk factor profiles: Comparative risk factor analysis of, drugs, and over twelve comorbidities years. Endocrine. 2016;54(1): 241-255.

5. Chua JR, Tee ML. Association of sarcopenia with osteoporosis in patients with chronic obstructive pulmonary disease. Osteoporosis and sarcopenia. 2020;6(3): 129-132.

6. Kim JE, Lee YH, Huh JH, Kang DR, Rhee Y, Lim SK. Early-stage chronic kidney disease, osteoporosis, and as risk factors of insulin resistance in population aged: the fourth Korea National Health and Nutrition Examination Survey (KNHANES IV), 2008-2009. international Osteoporosis: a established journal as result of the between Foundation European for Bone loss and the Bone loss USA Foundation National of the. 2015;25(9): 2189-2198.

7. Saeki C, Oikawa T, Kanai T, et al. Relationship between osteoporosis, sarcopenia, fracture vertebral, and sarcopenia in patients with biliary primary cholangitis. European journal of gastroenterology \& 
hepatology. 2021;33(5): 731-737.

8. Pan Y, Xu J. Association Between Muscle Mass, Bone Mineral Density and Osteoporosis in type 2 diabetes. Journal of diabetes investigation. 2021.

9. Osaka T, Hamaguchi M, Hashimoto $Y$, et al. Decreased the creatinine to cystatin $C$ surrogate marker sarcopenia ratio is a of in with patients T2DM. Res Diabetes Clin Pract. 2018;139: 52-58.

10. Gojanovic M, Holloway-Kew KL, Hyde NK, et al. The Dietary Inflammatory Index Is Associated with Low Muscle Mass and Low Muscle Function in Older Australians. Nutrients. 2021;13(4).

11. Kanis JA. Assessment of fracture risk and its application to screening for postmenopausal osteoporosis: synopsis of a WHO report. WHO Study Group. Bone loss international: a journal as established lead to between the Foundation European for Osteoporosis and the National Osteoporosis Foundation of the USA. 1994;4(6): 368-381.

12. Yang J, Zhang T, Feng D, et al. A new diagnostic index for sarcopenia and its relationship short with term post-operative patients in surgery undergoing for colorectal cancer. Disease Colorectal: the official journal of the Association of Coloproctology of Great Britain and Ireland. 2019;21(5): 538547.

13. Lin YL, Chen SY, Lai YH, et al. Serum creatinine to cystatin $C$ ratio predicts muscle skeletal strength and mass in dialysis patients with kidney disease chronic. Clinical nutrition. 2020;39(8): 2435-2441.

14. Yanishi $M$, Kinoshita $H$, Tsukaguchi $H$, et al. The creatinine/cystatin $C$ ratio gives evaluation effective of mass muscle in transplant recipients kidney. International urology and nephrology. 2019;51(1): 79-83.

15. Hirai K, Tanaka A, Homma T, et al. Serum creatinine/cystatin $\mathrm{C}$ ratio as a surrogate sarcopenia patients marker for in with chronic obstructive disease pulmonary. Clinical nutrition. 2021;40(3): 1274-1280.

16. Fu X, Tian Z, Wen $S$, et al. A new index based on serum creatinine and cystatin $C$ useful is assessing for in advanced sarcopenia patients cancer. Nutrition. 2020;82: 111032.

17. Lu YW, Tsai YL, Chou RH, et al. Serum creatinine to cystatin $C$ ratio is associated adverse major with events cardiovascular in coronary artery obstructive patient disease. Nutrition, metabolism, and cardiovascular diseases: NMCD. 2021;31(5): 1509-1515.

18. Tabara Y, Okada Y, Ochi M, Ohyagi Y, Igase M. Association of Creatinine-to-Cystatin C Ratio with Myosteatosis and Physical Performance in Older Adults: The Japan Shimanami Health Promoting Program. Journal of the American Medical Directors Association. 2021.

19. Srikanthan P, Hevener AL, Karlamangla AS. Sarcopenia exacerbates obesity- resistance insulin associated findings and: dysglycemia the Nutrition from Examination Survey III and National Health. PloS one. 2010;5(5): e10805.

20. Landi F, Cruz-Jentoft AJ, Liperoti R, et al. Sarcopenia and mortality risk in frail persons aged older years 80 older and: from study.ilSIRENTE ageing and Age. 2013;42(2): 203-209.

21. Liu W, Zhu X, Tan X, et al. Predictive Value of Serum Creatinine/Cystatin C in Acute Ischemic Stroke Patients under Nutritional Intervention. The journal of nutrition, health \& aging. 2021;25(3): 335-339. 
22. Qiu S, Cai X, Yuan Y, Xie B, Sun Z, Wu T. Changes in creatinine-to-cystatin C 4 years over ratio, diabetes risk, and control of cardiometabolic: The Health and China Retirement Longitudinal Study. Journal of diabetes. 2021.

23. Zofkova I. Hormonal aspects of the muscle-bone unit. Physiological research. 2008;57 Suppl 1: S159-169.

24. Macdonald $\mathrm{H}$, Kontulainen $\mathrm{S}$, Petit $\mathrm{M}$, Janssen $\mathrm{P}, \mathrm{McKay} \mathrm{H}$. Bone strength and its determinants in preand early pubertal boys and girls. Bone. 2006;39(3): 598-608.

25. Lang TF. The bone-muscle relationship in men and women. Journal of osteoporosis. 2011;2011: 702735.

26. Hsu CY, Chen LR, Chen KH. Osteoporosis in Patients with Chronic Kidney Diseases: A Systemic Review. International journal of molecular sciences. 2020;21(18).

\section{Figures}




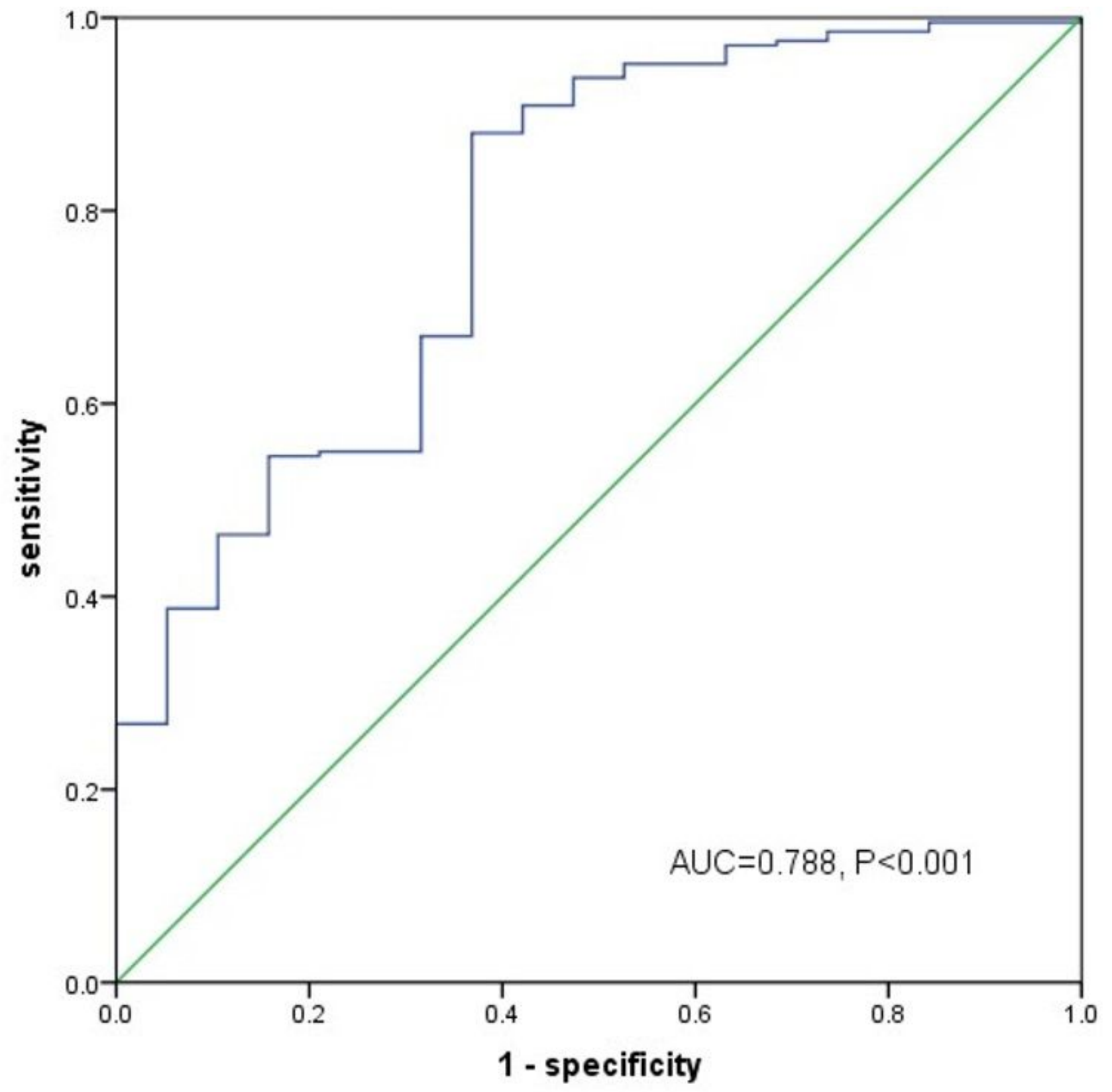

Figure 1

Receiver operating characteristic analysis of the CCR for osteoporosis among males 